Running head: Memory specificity, problem solving and eating psychopathology

\title{
The influence of eating psychopathology on autobiographical memory specificity and social problem-solving
}

\author{
Nathan Ridout* a , Munveen Matharu ${ }^{\mathrm{a}}$, Elizabeth Sanders ${ }^{\mathrm{a}}$ and Deborah J. Wallis ${ }^{\mathrm{b}}$ \\ ${ }^{a}$ Department of Psychology, School of Life \& Health Science, Aston University \\ ${ }^{\mathrm{b}}$ Loughborough University Centre for Research into Eating Disorders, School of Sport \\ Exercise and Health Sciences, Loughborough University
}

\begin{abstract}
Address for correspondence*
Dr Nathan Ridout

Department of Psychology,

School of Life \& Health Science,

Aston University

Birmingham, UK
\end{abstract}

B4 7ET

Tel: +44 (0) 1212044162

Fax: +44 (0) 1212044090

Email: n.ridout@aston.ac.uk

This is the accepted version of the manuscript published by Elsevier in Psychiatry Research, doi:10.1016/j.psychres.2015.06.030

\section{$\underline{\text { https://doi.org/10.1016/j.psychres.2015.06.030 }}$}

Ridout, N., Matharu, M., Sanders, E., \& Wallis, D.J. (2015). The influence of eating psychopathology on autobiographical memory specificity and social problem-solving.

Psychiatry Research, 228, 295-303. 


\begin{abstract}
The primary aim was to examine the role of mood and eating psychopathology on autobiographical memory specificity (AMS) and social problem solving (SPS). A further aim was to establish if AMS mediated the relationship between eating psychopathology and SPS. A non-clinical sample of 52 female undergraduates completed the autobiographical memory test (AMT), where they were asked to retrieve specific memories of events from their past in response to emotional cue words, and the meansend problem-solving task (MEPS), where they were asked to generate means of solving a series of social problems. Participants also completed the Eating Disorders Inventory (EDI) and Hospital Anxiety and Depression Scale. After controlling for mood, high scores on the EDI subscales, particularly Drive-for-Thinness, were associated with the retrieval of fewer specific and a greater proportion of categorical memories on the AMT. High EDI scores were also associated with the generation of fewer and less effective means on the MEPS. Furthermore, AMS fully mediated the relationship between eating psychopathology and SPS performance. The current findings have implications for individuals exhibiting high levels of eating psychopathology, as poor AMS and SPS are likely to impact negatively on their long term mood, psychological wellbeing and everyday social functioning.
\end{abstract}

Key words:

Overgeneral memory; social functioning; eating disorders; depression; anxiety 


\section{Introduction}

Autobiographical memory (AM) refers to the recollection of personally experienced events from the past. This aspect of memory is central to an individual's experience of the self and is vital for successful goal attainment (Conway \& Pleydell-Pearce, 2000). There is convincing evidence that information in autobiographical memory is organised hierarchically, with general representations of events leading to more specific and detailed representations. This relates to an important element of goal pursuit, the ability to retrieve memories in sufficient detail for the task in hand. There is considerable evidence, using word cuing paradigms like the autobiographical memory test (AMT; Williams \& Broadbent, 1986), that certain groups, notably patients with depression and/ or post-traumatic stress disorder (PTSD), have impaired access to specific representations of personal events (see Williams et al., 2007 for a review). For example, if presented with the cue word "Party", these individuals would be more likely to respond with a general memory "I used to enjoy parties when I was younger" rather than a specific event "I went to Emily's birthday party in February". In addition to the findings of impaired autobiographical memory specificity in patients with clinical depression there is a growing body of evidence demonstrating similar deficits in participants with subclinical depression, referred to as dysphoria (Dalgleish et al., 2007; Dickson \& Bates, 2006; Goddard, Dritschel \& Burton, 1997; Ramponi, Barnard \& Nimmo-Smith, 2004). Some studies have also reported a negative relationship between AMS and measures of depression severity (e.g. Dalgleish et al., 2007; Drummond et al., 2006, Johannessen \& Berntsen, 2009; Kaviani et al., 2011). Further evidence of the influence of mood on memory specificity comes from a study conducted by Au Yeung, Dalgleish, Golden \& Schartau (2006) that demonstrated impaired memory specificity in never-depressed participants following a negative mood induction. The decrease in AMS correlated with the reduction of self-rated happiness. Taken together there is clear evidence that mood, particularly depression, can influence memory specificity. However, this tendency does not generalise to anxiety, as there is no evidence of AMS deficits in patients with generalised anxiety disorder (Burke \& Mathews, 1992; Wessel et al., 2001) and in nonclinical participants with high trait anxiety (Richards \& Whittaker, 1990). 
As noted by Dalgleish et al. (2007) deficits in autobiographical memory specificity (AMS) are more than a mere cognitive curiosity; rather, it appears to be closely linked to processes relating directly to the onset, maintenance and recovery from a number of psychopathological conditions. Indeed, there is a growing body of evidence that impaired AM retrieval is associated with a number of negative consequences. For example, the overgeneral memory style is related to the persistence of symptoms in depressed patients (Brittlebank, Scott, Williams, \& Ferrier, 1993; Dalgleish et al., 2001 and Peeters et al., 2002; See Sumner et al., 2010 for a meta-analysis). Poor autobiographical memory specificity (AMS) also predicts negative mood in nondepressed participants in response to challenging life events (Gibbs \& Rude, 2004; Mackinger, Loschin, \& Leibetseder, 2000a; Mackinger, Pachinger, Leibetseder, \& Fartacek, 2000b; van Minnen, Wessel, Verhaak, \& Smeenk, 2005) and has been shown to moderate the impact of daily hassles on depression, such that chronic life stress had a greater impact on the mood of individuals with poorer memory specificity (Anderson, Goddard \& Powell, 2010). Evidence has demonstrated that AMS is negatively related to feelings of hopelessness (Arie et al., 2008; Kaviani et al., 2011), which in turn is a significant predictor of suicide and parasuicidal behaviour (Victor \& Klonsky, 2014). Deficits in AMS are also associated with impaired social problem solving (Evans, Williams, O’ Loughlin \& Howells, 1992). Goddard, Dritschel and Burton (1996) assessed clinically depressed patients on the AMT and the means-end problem solving task (MEPS; Platt and Spivack, 1975). The MEPS involves presenting participants with hypothetical social scenarios and asking them to generate means of getting from the initial state (e.g. you realise you best friend is not talking to you) to the end goal state (you and your friend are talking again). The number of valid means generated and the effectiveness of these means (experimenter rated) indicate the quality of social problem solving. Goddard et al. (1996) reported that depressed patients exhibited reduced AMS and generated fewer, and less effective, means on the MEPS. Patients recalling fewer specific memories also generated fewer means on the MEPS, and their means were less effective than those generated by healthy controls. The authors also highlighted that retrieval of categorical memories during the MEPS was particularly detrimental to performance (fewer and less effective means). These findings have been replicated in 
other samples of clinically depressed patients (Raes et al., 2005), bipolar patients (Scott et al., 2000) and participants with subclinical depression (Goddard, Dritschel \& Burton, 1997). Importantly, it has been shown that autobiographical memory specificity mediates the relationship between negative mood and problem-solving performance (Williams, Barnhofer, Crane, \& Beck, 2005). Further evidence of the link between autobiographical memory specificity and social problem solving comes from studies 4 and 5 of Eade et al. (2006), who demonstrated that directly manipulating participant's memory specificity impacted on their performance on the MEPS.

There is emerging evidence that individuals with eating disorders (ED) also demonstrate reduced autobiographical memory specificity (AMS). For example, Dalgleish et al. (2003) assessed a mixed sample of patients with anorexia nervosa (AN) and bulimia nervosa $(\mathrm{BN})$ on the AMT and reported that, in comparison to a healthy control group, the patients with ED recalled fewer specific memories. This finding has subsequently been replicated in homogenous samples of patients with AN (Brockmeyer, Holtforth, Bents, Herzog, \& Friederich, 2013; Nandrino, Doba, Lesne, Christophe \& Pezard, 2006 and Kovacs, Szabo \& Paszthy, 2011) and BN (Laberg \& Andersson, 2004). It is important to note that these findings were independent of the influence of depression on memory specificity. Laberg and Andersson (1994) also reported that the AMS deficit was evident in patients that were in remission from their ED. This is consistent with findings showing that poor AMS persists in remitted depressed patients (Mackinger et al., 2000a; 2000b; Williams \& Dritschel, 1988). Reduced AMS has also been reported in non-clinical participants who might be considered 'at risk' of developing eating disorders. Ball, Singer, Kemps and Tiggemann (2010) reported that individuals scoring high on the concern-with-dieting subscale of the restraint scale (Herman et al., 1978) exhibited impaired retrieval of specific memories on the AMT. However, they did not find a specificity difference between dieters and non-dieters, which is inconsistent with the findings of Johannessen and Berntsen (2009). As noted by Ball et al. (2010), Despite these studies it has yet to be established if participants reporting subclinical levels of eating psychopathology, as measured by validated measures such as the Eating Disorders Inventory (EDI; Garner, Olmstead \& Polivy, 1983) would exhibit deficits in autobiographical memory retrieval. The current study represents the first 
attempt to establish if deficits in memory specificity are evident in participants with subclinical eating psychopathology.

In addition to reported memory specificity deficits, there is also evidence that eating disorders are associated with impaired social problem-solving. For example, Grissett and Novell (1992) reported that patients with bulimia self-reported problems in social situations (on the Social Competence Scale; Sarason et al., 1985) and were rated, by independent observers (blind to their group membership), as less effective in a social context than were healthy controls. Holt and Espelage (2002) utilised a measure, the Anorexia and Bulimia Problem-solving Inventory (ABPI; Espelage et al., 2000), that was specifically designed to assess problem-solving in an eating disorders context and reported that participants indicating significant eating psychopathology on the EAT inventory exhibited less effective social problem solving than did healthy controls. This was particularly evident in scenarios relating to eating, weight, and interpersonal relationships. More recently, Swanson et al. (2010) reported that patients with AN exhibited maladaptive problem solving skills on the social problem solving inventory revised (SPSI-R; D’Zurilla \& Maydeu-Oliveres, 1995). Thus far, research into social functioning in eating disorders has relied on self-report measures such as the SPSI-R, it has yet to be established if participants with eating psychopathology would exhibit deficits on an independently-assessed outcome measure of social problem-solving, such as the MEPS. Therefore, this study is the first to utilise an objective measure of social problem solving to investigate social functioning in participants with disordered eating.

To date, social problem solving has not been examined in non-clinical participants with elevated scores on measures of eating psychopathology. Furthermore, the role of AMS in social problem solving has yet to be determined in individuals with disordered eating. Therefore, the aim of the current study was to examine to role of mood and eating psychopathology on autobiographical memory specificity (AMS) and social problem solving. A further aim was to establish if AMS mediated the relationship between eating psychopathology and social problem solving (see Figure 1). To that end, a non-clinical sample of participants was assessed on the standard AMT, MEPS and measures of mood and disordered eating attitudes: Hospital Anxiety and Depression Scale (HADS; Zigmond \& Snaith, 1983) and Eating Disorders Inventory (EDI; Garner, 1991) respectively. We 
predicted that, after controlling for mood, the proportion of specific memories retrieved on the AMT would be negatively related to scores on the EDI subscales. Furthermore, we predicted that, after controlling for mood, the number and effectiveness of means on the MEPS would be negatively related to scores on the subscales of the EDI. We also expected that the number and effectiveness of means on the MEPS would be positively related to the proportion of specific memories and negatively related to the proportion of categorical memories retrieved on the AMT. Finally we expected that AMS would mediate the relationship between eating psychopathology and social problem solving performance. The proposed mediational model is presented in Figure 1. We expected that the direct relationship between eating psychopathology and social problem solving (c') would become less significant (partial mediation) or non-significant (full mediation) after controlling for the effect of AMS on social problem solving. This would provide evidence that eating psychopathology influences social problem solving indirectly $(a+b)$ by reducing AMS (and/ or increasing categorical retrieval), which in turn impacts on SPS.

[Insert Figure 1 about here]

\section{Method}

\subsection{Participants}

52 female undergraduate students, recruited using Aston University's research participation scheme, took part in the study for course credit. The mean age of the participants was 20.1 years $(\mathrm{SD}=2.4)$. Participant characteristics are shown in Table 1. All participants self-reported that they were medication free and had no history of treatment for eating disorders ${ }^{1}$, depression or anxiety. The majority of reported BMI scores are within normal range ${ }^{2}$.All participants gave full written informed consent prior

\footnotetext{
${ }^{1}$ Although all participants self-reported no history of an eating disorder; $8 / 52$ participants (15\% of the sample) reported EDI scores in the same range as the clinical groups used to validate the EDI (Garner et al., 1984), suggesting that they might meet diagnostic criteria for an eating disorder. This was controlled for during analysis of the memory and social problem solving data.

${ }^{2}$ BMI scores were only collected from 16 participants. However, the samples with and without BMIs did not differ in their scores on the EDI subscales, thus, given the evidence of significant relationships between BMI and scores on the EDI subscales (Meltzer et al., 2001; Wassenaar et al, 2000), there is no reason to suspect that the two samples would have differed significantly in their BMIs.
} 
to taking part in the study. The study was approved by Aston University's Research Ethics Committee.

\subsection{Materials and Measures}

2.2.1. Screening questionnaire: Current and/or prior treatment for depression, anxiety and/ or eating disorders was established using a simple screening questionnaire. Participants were asked to indicate if they had previously been treated for any of the above disorders and, if so, on how many occasions and when the last occasion occurred. Participants were also asked to indicate if they were currently being treated with any medication.

2.2.2. The eating and weight-related subscales of the Eating Disorders InventoryII (EDI-II; Garner, 1991): The 23 items making up the three eating and weight-related subscales of EDI-II (drive-for-thinness, bulimia and body dissatisfaction) were used to assess participants' attitudes to eating. These items focus on psychological and behavioural traits that are associated with eating disorders such as anorexia nervosa (AN) and bulimia nervosa (BN). Drive-for-thinness (DFT) measures preoccupations with dieting, the desire to be thin, and fears about gaining weight. Body dissatisfaction (BD) assesses the degree to which participants are unhappy with their body and the bulimia (B) subscale concerns binge eating and purging behaviour. Each item consists of a statement, e.g. "I eat when I am upset" followed by a 6-point Likert scale (ranging from always to never). Participants circle the response on each item that best represents their behaviour and attitudes. Each item is scored such that the three most disordered responses in order of descending severity being scored 3,2 and 1 respectively and the remaining three responses being given a score of zero. Thus scores range from 0-21 on the drive-forthinness (DFT) and bulimia (B) subscales and from 0-27 on the body dissatisfaction (BD) subscale, with higher scores indicating greater eating psychopathology. These subscales have been shown to have construct validity (Garner, 1991; confirmed by Espelage et al. 2003) and to be highly reliable in student populations; for example Spresser, Keune, Filion and Lundgren (2012) reported alpha coefficients of .91 for BD, .90 for DFT, and .82 for Bulimia. 


\subsubsection{Hospital Anxiety Depression Scale (HADS; Zigmond and Snaith, 1983):}

The HADS is a 14 item questionnaire that is designed to identify the presence and severity of anxiety and depression. This measure comprises seven items relating to depressive symptoms and seven concerning anxiety. Each item consists of four statements relating to a particular symptom and participants are asked to indicate the statement that best represents how they have been feeling during the last week (including the day of testing). The statements on each item are scored 3, 2, 1 or zero in order of descending severity of depression or anxiety. Thus, scores on both subscales range from 0-21, with higher scores indicating greater depression or anxiety. This measure has been shown to be valid and reliable in a student population (e.g. Andrews, Hejdenberg \& Wilding, 2006 reported Cronbach's alpha of .82 for anxiety and .74 for depression) and was included in the current study to allow for the effects of mood on memory specificity and social problem-solving to be controlled.

\subsubsection{Autobiographical Memory Test (AMT; Williams \& Broadbent, 1986): The} ability of the participants to retrieve memories of specific events from their past was established using the traditional AMT. Participants were presented with a series of ten emotional words, drawn from William and Broadbent (1986), and were asked to retrieve a specific memory in response to each cue as quickly as possible. A specific memory was defined as "a memory of an event that occurred at a particular time and place and that lasted less than a day". The five positive cues were: happy, relieved, proud, eager and sunny and the five negative cues were: guilty, hopeless, failure, ugly and worse. The words were presented via a computer screen in an alternating fashion - one positive then one negative then another positive and so on. The words remained on screen for two seconds and participants were given a maximum of 60 seconds to recall a memory in response to each cue. The participant's descriptions of the memories were audio-recorded for later transcription and specificity rating. Two practice words were given prior to the main set of trials and feedback on performance on these two trials was provided to allow participants to understand the task. The main dependent variable of interest was the proportion of specific memories retrieved (controlling for the number of omissions), but the proportion of each type of retrieval error was also calculated. Categorical memories 
refer to summaries of repeated events (e.g. going to clubs, eating out in restaurants); extended memories refer to events lasting longer than a day (e.g. a holiday in the south of France); and semantic associates are not memories but items, objects, places or people that the participant may have associated with the cue (e.g. "my dad" in response to the cue 'proud'). Failures to retrieve a memory within 60 seconds or decisions not to respond to a particular cue are both scored as omissions. Memories were rated for specificity by two of the authors (MM and ES). A sample of 100 memories was rated by the principal author and there was a high level of inter-rater reliability $\kappa=.90$ and $\kappa=.87$ respectively.

\subsubsection{Means- End Problem Solving Task (MEPS; Platt and Spivack, 1975):}

Participants were presented with four scenarios relating to social interactions within the contexts of employment, friendship and interpersonal relationships. The scenarios were presented in written format with the initial state (e.g. "you realise that your best friend is not talking to you") and the end state (e.g. "your friend is talking to you again") of the problem clearly stated. Participants were then requested to form an ideal strategy, including indicating the steps that they would take, to get from the initial to the end state. Participants were initially provided with a practice problem and feedback on their performance in order to ensure that they understood the task before they were given the main set of four problems. The number of relevant means (i.e., instrumental steps that allow the respondent to move toward the goal) that were generated for each problem was the primary dependent variable of interest on the MEPS. A mean was scored "for each discrete step which is effective in enabling the hero of the story to reach the resolution stage of the story or to overcome an obstacle preventing the hero from reaching the goal in the story" (Platt \& Spivack, 1975 p.21). The effectiveness of the generated means was independently assessed by the researchers, who rated the effectiveness using a Likerttype scale ranging from 0 (not at all effective) to 7 (very effective) for each problem. Following the procedure of Marx, Williams and Claridge (1992), "a problem-solving strategy was considered effective if it maximised positive and minimised short- and longterm negative consequences, both personally and socially" (p.80). These ratings were then summed across the four problems to provide an overall measure of effectiveness. A greater number of means and higher effectiveness scores indicate more effective SPS. In 
order to confirm the reliability of experimenter ratings on the MEPS, NR rated a sample of 40 problems and Pearson correlation coefficients revealed that there was good interrater reliability for number of means (.87) and effectiveness (.84).

\subsection{Procedure}

Once participants had provided full written informed consent they were assessed on the AMT and the MEPS, the order of which was fully counterbalanced across all participants. Following these tasks, participants completed the screening, HADS and EDI-II questionnaires.

\section{Results}

\subsection{Autobiographical memory - proportion of specific memories}

Analysis revealed no significant difference in the proportion of specific memories retrieved in response to positive (Mean=.75, $\mathrm{SD}=.18)$ and negative cues $(\mathrm{M}=.78$, $\mathrm{SD}=.22) ; \mathrm{t}(51)=.81, \mathrm{p}>.05$. Therefore, the remaining analyses were conducted on total specificity scores collapsed across cue type. The total proportion of specific memories retrieved was significantly negatively related to scores on the HADS Anxiety subscale and scores on the Drive-for-Thinness (DFT), Bulimia and Body Dissatisfaction (BD) subscales of the EDI; r(52)=-.31, p<.05, r(52)=-.59, $\mathrm{p}<.001, \mathrm{r}(52)=-.38, \mathrm{p}<.01$ and $\mathrm{r}(52)=-$ $.40, \mathrm{p}<.01$ respectively. In order to determine the relative influence of these factors on the retrieval of specific memories a hierarchical regression was conducted with anxiety ratings entered at the first level and DFT, Bulimia and BD scores entered at the second level. These analyses revealed a significant model that accounted for around $9 \%$ of the variance in memory specificity $\left(\mathrm{R}^{2}=.09\right.$; adjusted $\left.\mathrm{R}^{2}=.08\right) ; \mathrm{F}(1,50)=5.25, \mathrm{p}<.05$. Anxiety entered as a significant predictor with a Beta of -.31, $\mathrm{p}<.05$. The second model was also significant and accounted for around $35 \%$ of the variance in memory specificity $\left(\mathrm{R}^{2}=.37\right.$; adjusted $\left.\mathrm{R}^{2}=.32\right) ; \mathrm{F}(4,47)=6.89, \mathrm{p}<.001$, which represented a significant increase in the amount of variance explained $\left(\mathrm{R}^{2} \Delta=.27\right) ; \mathrm{F}(3,47)=6.8, \mathrm{p}<.001$. DFT score entered as the only significant predictor; Beta $=-.51, \mathrm{p}<.05$.

\subsection{Autobiographical memory - proportion of retrieval errors}


The mean proportion of categorical memories (Mean=.08, $\mathrm{SD}=.13$ ) retrieved on the AMT was positively related to scores on all three subscales of the EDI; Drive-for-Thinness, Bulimia and Body Dissatisfaction (BD); $\mathrm{r}(52)=.54, \mathrm{p}<.001, \mathrm{r}(52)=.37, \mathrm{p}<.01$ and $\mathrm{r}(52)=.55, \mathrm{p}<.001$ respectively. Categorical retrieval was positively related to self-rated anxiety, $\mathrm{r}(52)=.3, \mathrm{p}<.05$, but not depression, $\mathrm{r}(52)=.25, \mathrm{p}>.05$. The mean proportion of extended memories $(\mathrm{M}=.13, \mathrm{SD}=.11)$ and omissions $(\mathrm{M}=.04, \mathrm{SD}=.09)$ was not significantly related to mood or scores on the EDI subscales, all tests $\mathrm{p}>.05^{3}$. In order to determine the relative influence of these factors on the retrieval of categorical memories a hierarchical regression was conducted with Anxiety ratings entered at the first level and DFT, BD and Bulimia scores entered at the second level. These analyses revealed a significant model that accounted for around $9 \%$ of the variance in positive specificity $\left(\mathrm{R}^{2}=.09\right.$; adjusted $\left.\mathrm{R}^{2}=.07\right) ; \mathrm{F}(1,51)=4.96, \mathrm{p}<.05$. Anxiety entered as a significant predictor with a Beta of $.30, \mathrm{p}<.05$. The second model was also significant and accounted for around $37 \%$ of the variance in memory specificity to positive cues $\left(\mathrm{R}^{2}=.27\right.$; adjusted $\left.\mathrm{R}^{2}=.22\right) ; \mathrm{F}(4,47)=6.9, \mathrm{p}<.01$, which represented a significant increase in the amount of variance explained $\left(\mathrm{R}^{2} \Delta=.28\right) ; \mathrm{F}(3,47)=7.0, \mathrm{p}<.001$. BD score entered as the only significant predictor; Beta $=-.32, \mathrm{p}<.05$.

\subsection{Means-End Problem Solving (MEPS)}

The average number of relevant means identified by the participants was $9.98(\mathrm{SD}=3.03)$ and the mean effectiveness rating was $16.12(\mathrm{SD}=3.99)$. The number of relevant means generated was significantly positively related to the proportion of specific memories retrieved on the AMT and negatively related to scores on the DFT subscale of the EDI; $\mathrm{r}(52)=.32, \mathrm{p}<.05$ and $\mathrm{r}(52)=-.27, \mathrm{p}=.053$. Hierarchical regression with AM specificity entered at the first step and scores on the DFT at the second step revealed a significant model that accounted for around $11 \%$ of the variance $\left(\mathrm{R}^{2}=.11\right.$; adjusted $\left.\mathrm{R}^{2}=.09\right)$ in the number of generated means; $\mathrm{F}(1,50)=6.04, \mathrm{p}<.05$. Specificity entered as significant predictor, Beta $=.33, \mathrm{p}<.05$. The second model accounted for $12 \%$ of the variance

\footnotetext{
${ }^{3}$ The retrieval error data were not normally distributed and attempts to normalise these data using transformations did not improve the distribution. Therefore, relationships were retested using nonparametric tests. The pattern of findings was identical, thus we report the results of the parametric analyses.
} 
$\left(\mathrm{R}^{2}=.12\right.$; adjusted $\left.\mathrm{R}^{2}=.08\right) ; \mathrm{F}(2,49)=3.32, \mathrm{p}<.05$, which did not represent a significant increase in the amount of variance explained $\left(\mathrm{R}^{2} \Delta=.009\right) ; \mathrm{p}>.05$. Neither variable (specificity or DFT score) entered as a significant predictor Beta $=.26$ and -.12 respectively; both tests $\mathrm{p}>.05$. The mean effectiveness rating was positively correlated with specificity on the AMT and negatively related to scores on the DFT subscale of the EDI; $r(52)=.33, p<.05$ and $r(52)=.29, \mathrm{p}<.05$ respectively. Hierarchical regression with specificity entered at the first step and DFT entered at the second step revealed a significant model that accounted for $11 \%$ of the variance $\left(\mathrm{R}^{2}=.11\right.$; adjusted $\left.\mathrm{R}^{2}=.08\right)$ in effectiveness ratings; $\mathrm{F}(1,50)=6.00, \mathrm{p}<.05$. Memory specificity entered as a significant predictor, Beta $=.33, \mathrm{p}<.05$. The second model accounted for $12 \%$ of the variance in MEPS effectiveness $\left(\mathrm{R}^{2}=.12\right.$; adjusted $\left.\mathrm{R}^{2}=.08\right) ; \mathrm{F}(2,49)=3.25, \mathrm{p}<.05$, which did not represent a significant increase in the amount of variance explained $\left(\mathrm{R}^{2} \Delta=.01\right), \mathrm{p}>.05$. Neither specificity nor DFT score entered as significant predictors of effectiveness in this model; Beta $=.25$ and -.13 respectively, $\mathrm{p}>.05$.

\subsection{Disordered eating, memory specificity and social problem solving}

A Sobel test revealed that autobiographical memory specificity (AMS) significantly mediated the relationship between eating psychopathology (Drive-for-Thinness score; DFT) and number of means on the social problem solving task; ZSobel=-2.26, $\mathrm{p}<.05$. There was a larger relationship between DFT score and MEPS performance when DFT scores were entered alone $($ Beta $=-.27, \mathrm{p}<.05)$ than when DFT was entered with AMS (Beta=-.13, p>.05). This shows full mediation of eating psychopathology by autobiographical memory specificity. Similarly, a Sobel test revealed that AMS mediated the relationship between DFT and the effectiveness of social problem solving; ZSobel=2.2, $\mathrm{p}<.05$. The relationship between DFT and effectiveness was larger when DFT was entered alone $(-.28 ; \mathrm{p}<.05)$ than when DFT score was entered with AMS $(-.13, \mathrm{p}>.05)$, which shows full mediation of eating psychopathology by autobiographical memory specificity.

Goddard et al. (1996) identified that the tendency to retrieve categorical memories was more strongly associated with impaired SPS than was the proportion of specific. Therefore, we examined the relationships between eating psychopathology, categorical 
retrieval on the AMT and performance on the SPS task. The number of means generated on the MEPS was significantly correlated with the proportion of categorical memories retrieved on the AMT; r(52)=-.29, p<.05. Similarly, the effectiveness of means generated on the MEPS was negatively related to categorical retrieval; $r(52)=-.33, p<.05$. A Sobel test revealed that proportion of categorical memories retrieved on the AMT significantly mediated the relationship between eating psychopathology (Drive-for-Thinness score; DFT) and the number of means on the MEPS; ZSobel=-1.6, p<.05 (one-tailed). There was a larger relationship between DFT score and number of generated means on the MEPS when DFT scores were entered alone (Beta=-.27, $\mathrm{p}<.05$ ) compared to when DFT was entered with the proportion of categorical memories (Beta=-.20, p>.05). This shows that categorical retrieval fully mediated the effect of DFT on the number of means generated on the MEPS. Similarly, the proportion of categorical memories mediated the relationship between DFT score and the effectiveness score on the social problem solving task; ZSobel=-1.7, $\mathrm{p}<.05$. There was a larger relationship between DFT score and MEPS effectiveness score when DFT scores were entered alone (Beta=-.28, $\mathrm{p}<.05$ ) compared to when DFT was entered with the proportion of categorical memories (Beta=-.19, p>.05). This shows that categorical retrieval fully mediated the effect of DFT on MEPS effectiveness. However, a Z-test revealed that there was no significant difference in the relative strengths of the relationships between AMS and MEPS performance (means and effectiveness) and categorical retrieval and MEPS performance; $Z=-.01, p>.05$ and $Z=-$ $.22, \mathrm{p}>.05$ respectively ${ }^{4}$.

\section{Discussion}

The aim of the current study was to examine to role of mood and eating psychopathology on autobiographical memory specificity (AMS) and social problem solving (SPS). A further aim was to establish if AMS mediated the relationship between eating psychopathology and social problem solving.

On the autobiographical memory test (AMT) we predicted that, after controlling for mood, the proportion of specific memories retrieved would be negatively related to

\footnotetext{
${ }^{4}$ All data were reanalysed excluding the 8 individuals scoring in the clinical range on the EDI and all findings held, therefore we report the findings from the full data set.
} 
scores on the EDI subscales. This prediction was supported by our findings, as memory specificity was negatively related to scores on the EDI. Examination of the different subscales of the EDI revealed that Drive-for-Thinness (DFT) was identified as the best predictor of memory specificity. Also as expected, the proportion of categorical memories was related to scores on the EDI, with Body Dissatisfaction (BD) identified as the best predictor of this type of overgeneral memory. The finding of reduced AMS is consistent with previous studies looking at AMS in participants 'at risk' of eating disorders (Ball et al., 2010; Johannessen \& Berntsen, 2009) and in patients with clinically diagnosed eating disorders (Brockmeyer et al., 2013; Dalgleish et al., 2003; Kovacs et al., 2011; Laberg \& Andersson, 2004; Nandrino et al., 2006).

The current findings could be considered within the framework of the influential CaR-FA-X model of autobiographical memory and psychopathology (Williams et al. 2007). The first element of this model, capture and rumination (CaR), proposes that, in individuals with highly activated and elaborated negative self-representations (schema), the memory search gets 'captured' at the intermediate level of the hierarchy, where general conceptual processing predominates. In these individuals, the tendency to retrieve an overgeneral memory is greater when cues relate closely to self-relevant concerns held in these schema (Crane, Barnhofer, \& Williams, 2007). Furthermore, ruminating (repetitive abstract thinking) about these concerns increases the likelihood of the memory search being captured at the general conceptual level (Watkins \& Teasdale, 2001, 2004; Watkins et al., 2000). As there is evidence of negative self-schema in patients with eating disorders (Luck et al., 2005; Stein \& Corte, 2008) and in subclinical groups who could be considered at risk of developing an eating disorder (Morris, Goldsmith, Roll, \& Smith, 2001) a deficit in AMS would be expected. Furthermore, it would be anticipated that this deficit would be greater when memory cues were closely related to the content of the negative self-schema. In line with this notion, Ball et al. (2010) reported reduced AMS in restrained eaters when cued with words relating to concerns about weight and body image. However, as they did not include non-concernrelated cues it is difficult to clearly interpret their data in terms of CaR. Similarly, although the current findings provide evidence of reduced AMS in individuals with subclinical eating psychopathology in response to non-concern related words, it is 
plausible that this tendency would have been greater had concern-related cues been used. Nevertheless, the findings of Johannessen and Berntsen (2009) are inconsistent with the model, as they reported that although dieters exhibited impaired AMS in comparison to non-dieters, this deficit was not greater for concern-related cues relative to neutral.

The second element of the Car-FA-X model, functional avoidance (FA), proposes that individuals strategically truncate the memory search at the general conceptual level in the hierarchy in an attempt to avoid accessing specific memories of traumatic or painful emotional experiences (Williams et al., 2007). Given that eating disorders are associated with repeated attempts to avoid and moderate negative affect (Lampard et al., 2010; Luck et al., 2005) it is plausible that the AMS deficit observed in the current study could relate to attempts by those with elevated scores on the EDI to control negative affect (it is notable that these participants also reported higher anxiety and depression scores than did low EDI scorers). However, as the current study was not specifically designed to examine this proposal, such speculations must be considered with caution.

The final aspect of the CAR-FA-X model concerns executive function (X). There is considerable evidence that executive processes play a key role in the retrieval of specific event memories (Conway \& Pleydell-Pearce, 2001) and have been implicated in the AMS deficits shown in depressed patients (Dalgleish et al., 2007), patients with PTSD (Dalgleish et al., 2008) and older adults (Holland, Ridout, Geraghty \& Walford, 2012). Given that impaired executive function has been reported in patients with eating disorders (Allen et al., 2013; Kemps et al., 2006) and in participants who might be considered at risk of developing an eating disorder (Green et al., 2003; Kemps \& Tiggemann, 2005) it is plausible that the deficit in AMS observed in the current study might be a consequence of reduced executive function in those with elevated EDI scores. However, as we did not include measures of executive function in the current study this cannot be confirmed. Future work should aim to establish if the role of executive processes in the AMS deficits observed in eating disorders is consistent with that shown in depression and PTSD (Dalgleish et al. 2007; 2008).

When considering the effect of mood on AMS, the lack of relationship between depression and AMS observed in the current study is consistent with the majority of studies looking at AMS in depression, as it is usually the case in clinically depressed 
samples that AMS does not correlate with depression severity (see Williams et al., 2007 for a review). Similarly, there have been several studies reporting no relationship between depression severity and AMS in non-clinical populations (e.g. Raes, Pousset \& Hermans, 2004; Raes, Hermans, Williams \& Eelen, 2006). However, some studies have reported a negative relationship between AMS and measures of depression severity (e.g. Dalgleish et al., 2007; Drummond et al., 2006, Kaviani et al., 2011). Interestingly, our findings are inconsistent with Johannessen and Berntsen (2009) who reported that the reduced AMS observed in dieters was associated with depression severity. The current finding that AMS was negatively associated with anxiety score is unusual and inconsistent with previous studies reporting no AMS deficits in patients with generalised anxiety disorder (Burke \& Mathews, 1992; Wessel et al., 2001) and in non-clinical participants with high trait anxiety (Richards \& Whittaker, 1990). Given that this relationship (between anxiety and AMS) became non-significant once the relationship between Drive-for-Thinness score and AMS was taken into account, it would seem likely that it is a consequence of the relationship between anxiety and eating psychopathology 5 .

In line with the suggestion of Ball et al. (2010) it is plausible that reduced AMS might be be a risk factor for the development of eating disorders, but this requires longitudinal studies to confirm. Nevertheless, the evidence that individuals with subclinical disordered eating have impaired AMS does have significant implications for these individuals in terms of their mood and everyday functioning. For example, previous findings support that having an overgeneral autobiographical memory style is a risk factor for depression (Anderson et al., 2010; Gibbs \& Rude, 2004; Mackinger et al., 2000a; 2000b; van Minnen et al., 2005). Further, there is an established link between poor AMS and hopelessness (Kaviani et al., 2011), which in turn is a strong predictor of suicide and self-harm (Victor \& Klonsky, 2014). Therefore, it is plausible that impaired AMS might act as a risk factor for suicidal and self-harming behaviour that has been reported in patients with eating disorders (Preti et al. 2011). Impaired AMS would also be expected to have implications for these individuals' ability to solve problems.

\footnotetext{
${ }^{5}$ A Sobel test revealed that DFT completely mediated the relationship between anxiety and AMS; ZSobel=-2.57, $\mathrm{p}<.01$.
} 
In line with this notion, there is evidence from the current study that disordered eating impairs social problem solving, as, in line with our predictions, the number and effectiveness of means on the MEPS was negatively related to scores on the DFT subscale of the EDI. Nevertheless, there was only partial support for our hypothesis, as this relationship was not evident for scores on the Bulimia and Body Dissatisfaction scales. To our knowledge this is the first study to demonstrate impaired social problem solving in participants who could be considered 'at risk' of developing eating disorders. However, the current findings are consistent with studies in clinical eating disorders (Espelage et al., 2000; Grissett \& Novell, 1992; Holt \& Espelage; 2002 Swanson et al., 2010).

As expected, the number and effectiveness of means on the MEPS was positively related to the proportion of specific memories retrieved on the AMT. This is the first demonstration of this relationship in participants with disordered eating, but is wholly consistent with previous studies looking at AMS and SPS in clinically depressed participants (Evans et al., 1992, Goddard et al., 1996, Williams et al., 2005) and dysphoria (Goddard et al., 1997). Also as expected, there was clear evidence that AMS fully mediated the relationship between eating psychopathology and social problem solving performance. This is consistent with findings in depression (Williams et al., 2005).

As Goddard et al. (1996) reported that the tendency to retrieve categorical memories was potentially more important in understanding impaired SPS than was specific recall, we examined the role of categorical retrieval in explaining SPS in our own sample. The number of means generated on the MEPS was not related to the proportion of categorical memories retrieved, but the ratings of effectiveness were negatively related to this tendency. Furthermore, categorical retrieval was shown to fully mediate the effect of eating psychopathology on effectiveness of SPS. However, as a similar mediational effect was seen for the proportion of specific memories retrieved (just in the opposite direction) it cannot be concluded that a tendency towards categorical retrieval was more detrimental to SPS than poor AMS. This is also confirmed by a Z-test demonstrating that there was no significant difference in the relative strengths of the correlations between AMS and effectiveness and categorical retrieval and effectiveness. However, following 
the logic of Goddard et al. (1997) it is plausible that specific retrieval aids SPS effectiveness by preventing categorical retrieval.

In sum, the current study provides clear evidence that, within a non-clinical sample, individuals with elevated scores on a measure of eating psychopathology exhibit impaired autobiographical memory specificity (AMS) and social problem solving (SPS). Further, our data demonstrate that the deficits in SPS were explained by the impairment in AMS. The deficit in AMS could have significant negative consequences in terms of greater vulnerability to depression and self-harm and poorer interpersonal functioning. It is also plausible that reduced AMS could play a role in the development of eating disorders. For example, via its impact on social functioning, it could lead to a weakening of social support. However, this needs further work to confirm. The growing evidence of impaired AMS in eating disorders suggests that targeted interventions to improve this aspect of memory, akin to those used in depressed patients (Raes et al., 2009), might be a useful adjunct to cognitive treatments for eating disorders. 


\section{References}

Allen, K.L., Byrne, S.M., Hii, H., van Eekelen, A., Mattes, E. \& Foster, J.K. (2013) Neurocognitive functioning in adolescents with eating disorders: A population-based study. Cognitive Neuropsychiatry; 18 (5), 355-375

Anderson, R. J., Goddard, L., \& Powell, J. H. (2010) Reduced specificity of autobiographical memory as a mediator of the relationship between daily hassles and depression. Cognition \& Emotion, 24(4), 702-709

Arie, M., Apter, A., Orbach, I., Yefet, Y., \& Zalzman, G. (2008). Autobiographical memory, interpersonal problem solving, and suicidal behavior in adolescent inpatients. Comprehensive Psychiatry, 49(1), 22-29.

Ball, C. T., Singer, S., Kemps, E., \& Tiggemann, M. (2010) Restrained eating and memory specificity. Appetite 55, 359-362

Brittlebank, A. D., Scott, J., Williams, J. M. G., \& Ferrier, I. N. (1993).Autobiographical memory in depression: State or trait marker? British Journal of Psychiatry, 162, 118121.

Brockmeyer, T., Skunde, M., Wu, M., Bresslein, E., Rudofsky, G., Herzog, W., et al. (2014). Difficulties in emotion regulation across the spectrum of eating disorders. Compr Psychiatry, 55(3), 565-571.

Burke, M., \& Mathews, A. (1992). Autobiographical memory and clinical anxiety. Cognition \& Emotion, 6, 23-35.

Conway, M. A., \& Pleydell-Pearce, C. W. (2000). The construction of autobiographical memories in the self-memory system. Psychological Review, 107, 261-288. 
Crane, C., Barnhofer, T., \& Williams, J. M. G. (2007). Cue self-relevance affects autobiographical memory specificity in individuals with a history major depression. Memory, 15, 312-327.

Dalgleish, T., Tchanturia, K., Serpell, L., Hems, S., Yiend, J., De Silva, P.,et al. (2003). Self-reported parental abuse relates to autobiographical memory style in patients with eating disorders. Emotion, 3, 211-222.

Dalgleish, T., Spinks, H., Yiend, J., \& Kuylen, W. (2001). Autobiographical memory style in seasonal affective disorder and its relationship to future symptom remission. Journal of Abnormal Psychology, 110, 335-340.

Dalgleish, T., Rolfe, J., Golden, A. M., Dunn, B. D., \& Barnard, P. J. (2008). Reduced autobiographical memory specificity and posttraumatic stress: exploring the contributions of impaired executive control and affect regulation. Journal of Abnormal Psychology, $117(1), 236-241$.

Dalgleish, T., Williams, J. M., Golden, A. M., Perkins, N., Barrett, L. F., Barnard, P. J., et al. (2007). Reduced specificity of autobiographical memory and depression: the role of executive control. Journal of Experimental Psychology: General, 136(1), 23-42.

Drummond, L. E., Dritschel, B., Astell, A., O'Carroll, R. E., \& Dalgleish, T. (2006). Effects of age, dysphoria, and emotion-focusing on autobiographical memory specificity in children. Cognition \& Emotion, 20(3-4), 488-505.

D’Zurilla, T. J., \& Maydeu-Olivares, A. (1995). Conceptual and methodological issues in social problem-solving assessment. Behavior Therapy, 26, 409-432.

Evans, J., Williams, J. M. G., O’Loughlin, S., \& Howells, K. (1992). Autobiographical memory and problem-solving strategies of parasuicide patients. Psychological Medicine, 22, 399-405. 
Espelage, D. L., Quittner, A. L., Sherman, R., \& Thompson, R. (2000). Assessment of problematic situations and coping strategies in women with eating disorders: Initial validation of a situation-specific problem inventory. Journal of Psychopathology and Behavioral Assessment, 22(3), 271-297.

Garner, D. M. (1991). Eating Disorder Inventory-2. Professional manual. Odessa, FL: Psychological Assessment Resources.

Gibbs, B. R., \& Rude, S. S. (2004). Overgeneral autobiographical memory as depression vulnerability. Cognitive Therapy and Research, 28(4), 511-526.

Goddard, L., Dritschel, B., \& Burton, A. (1996). Role of autobiographical memory in social problem solving and depression. Journal of Abnormal Psychology, 105(4), 609616.

Goddard, L., Dritschel, B., \& Burton, A. (1997). Social problem solving and autobiographical memory in non-clinical depression. British Journal of Clinical Psychology, 36, 449-451.

Green, M. W., Jones, A. D., Smith, I. D., Cobain, M. R., Williams, J. M. G., Healy, H., et al. (2003). Impairments in working memory associated with naturalistic dieting in women: No relationship between task performance and urinary 5-HIAA levels. Appetite, $40,145-153$.

Grissett, N. I. \& Norvell, N. K. (1992) Perceived social support, social skills, and the quality of relationships in bulimic women. Journal of Consulting and Clinical Psychology, 60, 293-299

Herman, C. R., Polivy, J., pliner, P., Threkeld, J., \& Munie, D. (1978). Distractibility in dieters and non-dieters. An alternative view of externality. Journal of Personality and Social Psychology, 36, 536-548. 
Holland, C. A., Ridout, N., Walford, E., \& Geraghty, J. (2012). Executive function and emotional focus in autobiographical memory specificity in older adults. Memory, 20(8), 779-793.

Holt, M. K., \& Espelage, D. L. (2002). Problem-solving skills and relationship attributes among women with eating disorders. Journal of Counselling and Development, 80(3), 346-354.

Johannessen, K. B., \& Berntsen, D. (2008). Motivation for weight loss affects recall from autobiographical memory in dieters. Memory, 17, 69-83.

Kemps, E., \& Tiggemann, M. (2005). Working memory performance and preoccupying thoughts in female dieters: Evidence for a selective executive impairment. British Journal of Clinical Psychology, 44, 357-366.

Kemps, E., Tiggemann, M., Wade, T., Ben-Tovim, D., \& Breyer, R. (2006). Selective working memory deficits in anorexia nervosa. European Eating Disorders Review, 14(2), 97-103.

Killen, J. D., Taylor, C., Haywood, C., Haydel, K., Wilson, D., Hammer, L., et al. (1996). Weight concerns influence the development of eating disorders. A 4-year prospective study. Journal of Consulting and Clinical Psychology, 64, 936-940.

Kovacs, T., Szabo, P., \& Paszthy, B. (2011). Reduced Specificity of Autobiographical Memory in Anorexia Nervosa. Journal of Cognitive and Behavioral Psychotherapies, $11(1), 57-66$.

Laberg, S. L., \& Andersson, G. (2004). Autobiographical memories in patients treated for bulimia nervosa. European Eating Disorders Review, 12, 34-41. 
Lampard, A. M., Byrne, S. M., McLean, N., \& Fursland, A. (2011). Avoidance of affect in the eating disorders. Eating Behaviors, 12(1), 90-93.

Luck, A., Waller, G., Meyer, C., Ussher, M., \& Lacey, H. (2005). The role of schema processes in the eating disorders. Cognitive Therapy and Research, 29(6), 717-732.

Mackinger, H. F., Loschin, G. G., \& Leibetseder, M. M. (2000). Prediction of postnatal affective changes by autobiographical memories. European Psychologist, 5, 52-61.

Mackinger, H. F., Pachinger, M. M., Leibetseder, M. M., \& Fartacek, R. R. (2000). Autobiographical memories in women remitted from major depression. Journal of Abnormal Psychology, 109, 331-334.

Morris, M. E., Goldsmith, T. E., Roll, S., \& Smith, J. E. (2001). A model for assessing the self-schemas of restrained eaters. Cognitive Therapy and Research, 25, 201-214.

Nandrino, J., Doba, K., Lesne, A., Christophe, V., \& Pezard, L. (2006). Autobiographical memory deficit in anorexia nervosa. Emotion regulation and effect of duration of illness. Journal of Psychosomatic Research, 61, 537-543.

Paterson, G., Power, K., Collin, P., Greirson, D., Yellowlees, A., \& Park, K. (2011). A Mediational Model of Self-Esteem and Social Problem-Solving in Anorexia Nervosa. European Eating Disorders Review, 19(2), 112-120.

Peeters, F., Wessel, I., Merckelbach, H., \& Boon-Vermeeren, M. (2002). Autobiographical memory specificity and the course of major depressive disorder. Comprehensive Psychiatry, 43, 344-350.

Platt, J. J., \& Spivack, G. (1975). Manual for the means-ends problem solving procedure. Philadelphia: Department of Mental Health Services, Hahnemann Community Mental Health/Mental Retardation Center. 
Preti, A., Rocchi, M. B. L., Sisti, D., Camboni, M. V., \& Miotto, P. (2011). A comprehensive meta-analysis of the risk of suicide in eating disorders. Acta Psychiatrica Scandinavica, 124(1), 6-17.

Raes, F., Pousset, G., \& Hermans, D. (2004). Correlates of autobiographical memory specificity in a non-clinical student population. Unpublished manuscript.

Raes, F., Hermans, D., Williams, J. M. G., Demyttenaere, K., Sabbe, B., Pieters, G., \& Eelen, P. (2005). Reduced specificity of autobiographical memories: A mediator between rumination and ineffective problem solving in major depression? Journal of Affective Disorders, 87, 331-335.

Raes, F., Hermans, D., Williams, J. M. G., \& Eelen, P. (2006). Reduced autobiographical memory specificity and affect regulation. Cognition \& Emotion, 20, 402-429.

Raes, F., Williams, J. M. G., \& Hermans, D. (2009). Reducing cognitive vulnerability to depression. A preliminary investigation of Memory Specificity Training in inpatients with depressive symptomatology. Journal of Behavior Therapy and Experimental Psychiatry, 40, 24-38.

Richards, A., \& Whittaker, T. M. (1990). Effects of anxiety and mood manipulation in autobiographical memory. British Journal of Clinical Psychology, 29, 145-153.

Scott, J., Stanton, B., Garland, A., \& Ferrier, I. N. (2000). Cognitive vulnerability in patients with bipolar disorder. Psychological Medicine, 30, 467-472.

Stein, K. F., \& Corte, C. (2008). The identity impairment model - A longitudinal study of self-schemas as predictors of disordered eating behaviors. Nursing Research, 57(3), 182190. 
Sumner, J. A., Griffith, J. W., \& Mineka, S. (2010). Overgeneral autobiographical memory as a predictor of the course of depression: A meta-analysis. Behav Res Ther, 48(7), 614-625.

Swanson, H., Power, K., Collin, P., Deas, S., Paterson, G., Grierson, D., et al. (2010). The Relationship Between Parental Bonding, Social Problem Solving and Eating Pathology in an Anorexic Inpatient Sample. European Eating Disorders Review, 18(1), $22-32$.

van Minnen, A., Wessel, I., Verhaak, C., \& Smeenk, J. (2005). The relationship between autobiographical memory specificity and depressed mood following a stressful life event: A prospective study. British Journal of Clinical Psychology, 44, 405-415.

Watkins, E., \& Teasdale, J. D. (2001). Rumination and overgeneral memory in depression. Effects of self-focus and analytical thinking. Journal of Abnormal Psychology,110, 353-357.

Watkins, E., \& Teasdale, J. D. (2004). Adaptive and maladaptive self-focus in depression. Journal of Affective Disorders, 82, 1-8.

Watkins, E., Teasdale, J. D., \& Williams, R. M. (2000). Decentring and distraction reduceovergeneral memory in depression. Psychological Medicine, 30, 911-920.

Wessel, I., Meeren, M., Peeters, F., Arntz, A., \& Merckelbach, H. (2001). Correlates of autobiographical memory specificity: The role of depression, anxiety and childhood trauma. Behaviour Research and Therapy, 39, 409-421.

Williams, J. M. G., Barnhofer, T., Crane, C., \& Beck, A. T. (2005).Problem solving deteriorates following mood challenge in formerly depressed patients with a history of suicidal ideation. Journal of Abnormal Psychology, 114, 421-431. 
Williams, J. M. G., \& Broadbent, K. (1986). Autobiographical memory in suicide attempters. Journal of Abnormal Psychology, 95, 144-149.

Williams, J. M. G., \& Dritschel, B. H. (1988). Emotional disturbance and the specificity of autobiographical memory. Cognition \& Emotion, 2, 221-234.

Williams, J. M.G., Barnhofer, T., Crane, C., Hermans, D., Raes, F., Watkins, E., \& Dalgleish, T. (2007) Autobiographical memory specificity and emotional disorder. Psychological Bulletin, 133, 122-148.

Zigmond, A. S., \& Snaith, R. P. (1983). The hospital anxiety and depression scale. Acta Psychiatrica Scandinavica, 67, 361-370. 
Table 1.

Participant characteristics

\begin{tabular}{lccc}
\hline & Mean & Standard Deviation & Range \\
\hline Age & 20.10 & 2.31 & $18-27$ \\
BMI* & 23.1 & 4.5 & $16-31$ \\
EDI - Drive for Thinness & 4.67 & 5.28 & $0-19$ \\
EDI - Bulimia & 1.79 & 2.43 & $0-11$ \\
EDI - Body Dissatisfaction & 9.83 & 7.38 & $0-27$ \\
Total EDI & 16.29 & 12.86 & $1-52$ \\
HADS - Anxiety & 7.90 & 4.11 & $1-17$ \\
HADS - Depression & 3.48 & 2.45 & $0-9$ \\
MEPS - Means & 9.98 & 3.03 & $2-18$ \\
MEPS - Effectiveness & 16.12 & 3.99 & $5-25$ \\
\hline
\end{tabular}

EDI=Eating Disorders Inventory; HADS=Hospital Anxiety and Depression Scale; MEPS=Means

End Problem-solving task. *BMI only collected from 16 participants. 
Table 2.

Autobiographical Memory Test: Mean proportion of retrieval errors (Standard deviations are presented in parentheses).

\begin{tabular}{lcc}
\hline Type of Retrieval Error & Mean & Standard Deviation \\
\hline Categorical & .08 & .13 \\
Extended & .13 & .11 \\
Semantic Associate & .002 & .02 \\
Omissions & .04 & .09 \\
\hline
\end{tabular}

